\title{
Evaluating Possibilistic Valid-Time Queries
}

\author{
Christophe Billiet $^{1}$, José Enrique Pons ${ }^{2}$, Olga Pons Capote ${ }^{2}$, and Guy De Tré ${ }^{1}$ \\ 1 Department of Telecommunications and Information Processing, Ghent University \\ Sint Pietersnieuwstraat 41, B-9000 Ghent, Belgium \\ Christophe.Billiet, Guy.DeTre@ugent . be \\ 2 Department of Computer Science and Artificial Intelligence, Escuela Técnica \\ Superior de Ingeniería Informática, Universidad de Granada \\ C/Periodista Daniel Saucedo Aranda s/n, E-18071 Granada, Spain \\ jpons, opc@decsai.ugr.es
}

\begin{abstract}
A temporal database schema models objects or concepts with time-related or time-variant properties and a derived database contains measurements or descriptions concerning these temporal properties. The modelling of temporal information in a temporal database has an impact on the consistency of the database. Of course, this temporal information can be queried. In some cases, information in the query's temporal demand is (partially) unknown. This paper presents a technique for querying a valid-time relation using, among others, a temporal constraint in which the time indication in the temporal expression contains uncertainty and a method for evaluating such queries. This should allow querying using a (partially) unknown temporal demand.
\end{abstract}

Keywords: valid-time database, uncertainty, ill-known intervals, uncertain temporal querying

\section{Introduction}

A database contains data representing real objects or concepts. Every one of these data is a measurement or description of a property of a real object or concept. In reality, some aspects or properties of objects or concepts are timevariant or time-related. E.g. the moment of a bank transaction is traditionally a moment in time and thus a time-related notion, the function of an employee in a company can change through recorded history and is thus time-variant. A temporal database schema [10] is a database schema that models real objects or concepts with time-related or -variant properties. However, the modelling of temporal aspects has a direct impact on the consistency of the temporal database, because the temporal nature of these aspects imposes extra integrity constraints. An example. Consider a relation in a relational library database, modelling the presence of books in the library. Every physical book is represented by a unique identifier. Every record in the relation contains such an identifier, a date on which the corresponding book was loaned and a date on which it was subsequently returned (if it is returned). Without further precautions, a library employee could 
add several records with the same book identifier, different 'loaned'-dates and no 'returned'-dates. This would represent a situation in which the same physical book was loaned several times on different dates and never returned, which is of course impossible. A temporal database schema will typically constrain record insertion and prevent similar modelling inconsistencies.

Humans handle temporal information using certain temporal notions like time intervals or time points [10]. The modelling and handling of these temporal notions in information systems has always been seen as a difficult task [3]. Klein [12] studied the concept of time in language and Devos [5] modelled vague temporal expressions by means of fuzzy sets [19]. Notably, temporal relationships between time intervals (and as a special case instants [10]) were studied by Allen [1]. Among many others, an issue in temporal modelling is the possible imperfection in (descriptions of) temporal notions. E.g. the temporal notion in a sentence like 'The Belfry of Bruges was finished on one single day somewhere between 1/01/1201 A.D. and 31/12/1300 A.D.' contains imperfection because of the uncertainty in the used time-related expression. It is known that the building was finished on a single day, but it is not known precisely which day this was.

To allow information systems to cope with these and similar imperfections, many approaches adopt fuzzy sets for the representation of temporal information [13], [14], [2], [6]. The temporal relationships studied by Allen were fuzzified by several authors [16], [14], [18].

Next to this, the inclusion of temporal indications in a relational database leads to several practical problems. In some specific works, authors consider the necessity of allowing imprecision in the representation of temporal indications [4], [11]. Most of these approaches use the concepts of fuzzy numbers and fuzzy intervals to represent imprecise temporal indications and considerable attention has thus been given to the problem of transforming two fuzzy numbers which represent the boundaries of an imprecise time interval into one fuzzy interval representing this interval. There are several proposals for this transformation, but some of them might show signs of minor issues, as pointed out in [17].

In this work, an approach is proposed that allows users to query a validtime relation using temporal demands containing uncertainty. The novelty of this approach is that it allows uncertainty in the query's temporal demand. The presented approach is based on the possibilistic framework for set evaluation found in [17]. In section 2, some main concepts are explained and the part of the framework on which this work is based, is briefly presented. In section 3, this paper's approach is presented. Finally, in section 4, conclusions are drawn and some directions for future research are presented.

\section{Preliminaries}

This section starts with a brief introduction to temporal databases. Next, some basic concepts are introduced, concerning possibilistic variables and ill-known values, sets and intervals. Last, the framework of set evaluation using ill-known constraints [17] is explained. 


\subsection{Temporal Databases}

As explained in the introduction, a temporal database schema models objects or concepts with time-related or -variant properties and a temporal database contains measurements or descriptions of temporal properties of these objects or concepts. Also, the modelling of temporal aspects has a direct impact on the consistency of the temporal database.

In [10], a consensus glossary on temporal terminology has been presented. Next, some of the important concepts from this glossary are described.

A chronon is a non-decomposable time interval with minimal duration and the database is thus unable to distinguish time intervals shorter than a chronon.

Based on their interpretation and modelling purpose, temporal data in a temporal database can be classified into four types. Of these types, user-defined time contains temporal data without impact on the consistency of the temporal database. These data are not handled specifically. The other types are:

- transaction time [10] contains temporal data describing when a fact is stored in the database and not yet logically deleted

- valid time [10] contains temporal data describing when a fact is true in the modelled reality

- decision time [15] contains temporal data describing when an event was decided to happen

Other possible types of database models are bi-temporal (both valid and transaction time) [10] or tri-temporal (valid, transaction and decision time) [15] models. To deal with descriptions of time points [9] or intervals [11] that are subject to imperfection, fuzzy temporal models [18] exist.

\subsection{Possibilistic Variables and Ill-known Values, Sets and Intervals}

In this section, possibilistic variables and the concepts of ill-known values, illknown sets and ill-known intervals are introduced, based on [17]. These concepts rely heavily on possibility theory [8] and its concepts like 'possibility' and 'possibility distribution'. In this work, 'possibility' is always interpreted as a measure of plausibility. A possibilistic variable is defined as follows [17].

Definition 1. A possibilistic variable $X$ on a universe $U$ is defined as a variable taking exactly one value in $U$, but for which this value is (partially) unknown. The variable's possibility distribution $\pi_{X}$ on $U$ models the available knowledge about the value that $X$ takes: for each $u \in U, \pi_{X}(u)$ represents the possibility that $X$ takes the value $u$. This possibility is interpreted as a measure of plausibility and thus as a measure of how plausible it is that $X$ takes the value $u$, given (partial) knowledge about the value $X$ takes.

Consider a set $R$ containing single values (and not collections of values). When a possibilistic variable $X_{v}$ is defined on such a set $R$, the unique value $X_{v}$ takes, which is (partially) unknown, is called an ill-known value in this work [7]. 
Now, consider a set $R$ containing single values and its powerset $\wp(R)$. When a possibilistic variable $X_{s}$ is defined on the powerset $\wp(R)$ of such a set $R$, the unique value $X_{s}$ takes will be a crisp set and the possibility distribution $\pi_{X_{s}}$ of $X_{s}$ will be a possibility distribution on $\wp(R)$. This $\pi_{X_{s}}$ will define the possibility of each value of $\wp(R)$ (a value of $\wp(R)$ is a crisp subset of $R$ ) being the value $X_{s}$ takes. This exact value $X_{s}$ takes, is called an ill-known set [7].

Consider a set $R$ containing single values and its powerset $\wp(R)$. Now consider a subset $\wp_{I}(R)$ of $\wp(R)$ and let this subset contain every element of $\wp(R)$ that is an interval, but no other elements. When a possibilistic variable $X_{i}$ is defined on the subset $\wp_{I}(R)$ of the powerset $\wp(R)$ of some set $R$, the unique value $X_{i}$ takes will be a crisp interval and the possibility distribution $\pi_{X_{i}}$ of $X_{i}$ will be a possibility distribution on $\wp_{I}(R)$. This $\pi_{X_{i}}$ will define the possibility of each value of $\wp_{I}(R)$ (a value of $\wp_{I}(R)$ is a crisp interval in $R$ ) being the value $X_{i}$ takes. This exact value the variable takes, is called an ill-known interval here.

In this work, another approach to defining and describing an ill-known interval is used. Here, an ill-known interval $I$ is defined and described by its start and end point, which are ill-known values. Thus, an ill-known interval is seen as an interval of which the exact start and end point are (partially) unknown, which implies that the interval itself is (partially) unknown. Thus, the start and end point of an ill-known interval are mutually independent ill-known values, which are defined by mutually independent possibilistic variables.

In intentions, both approaches are the same: they attempt to model a single interval for which some uncertainty exists about which values are in the interval and which are not. The approach mentioned first does this by defining the possibility that an interval is the meant interval, for every interval in the considered interval set, whereas the second approach describes the possibility of a single point being the start point of the meant interval and the possibility of a single point being the end point of the meant interval, for every point imaginable. The actual ill-known interval is then inferred from these start and end points.

Whatsoever, the interactions and behaviors of these representations and the correspondences, interactions and transformations between them are part of the current research of the authors.

A specific application of possibilistic variables is obtained when the universe under consideration is the set of boolean values, denoted $\mathbb{B}=\{T, F\}$, where $T$ denotes 'true' and $F$ denotes 'false' [17]. Indeed, any boolean proposition $p$ takes exactly one value in $\mathbb{B}$. If the knowledge about which value this proposition $p$ takes is given by a possibility distribution $\pi_{p}$, proposition $p$ can be seen as a possibilistic variable. As the interest lies with the case where the proposition holds (denoted $p=T$ ), the possibility and necessity that $p=T$ demand most attention. In this work, the following notations are used:

$$
\begin{aligned}
\text { Possibility that } p & =T: & & \operatorname{Pos}(p)=\pi_{p}(T) \\
\text { Necessity that } p & =T: & & \operatorname{Nec}(p)=1-\pi_{p}(F)
\end{aligned}
$$




\subsection{Interval Evaluation by Ill-known Constraints}

The problem of interval evaluation is more generally explained in [17]: basically, the need exists to check how all points in a crisp set are positioned with respect to one or more ill-known values.

In [17], the notion of an ill-known constraint is introduced:

Definition 2. Given a universe $U$, an ill-known constraint $C$ is specified by means of a binary relation $R \subseteq U^{2}$ and a fixed, ill-known value defined by its possibilistic variable $V$ on $U$, i.e.:

$$
C \triangleq(V, R)
$$

Some set $A \subseteq U$ now satisfies this constraint $C$ if and only if:

$$
\forall a \in A:(V, a) \in R
$$

An example of an ill-known constraint is $C_{<} \triangleq(X,<)$. Some set $A$ then satisfies $C_{<}$if $\forall a \in A: X<a$.

The satisfaction of a constraint $C \triangleq(V, R)$ by a set $A$ is basically a Boolean matter and can thus be seen as a boolean proposition, but due to the uncertainty inherent to the ill-known value $V$, it can be uncertain whether $C$ is satisfied by $A$ or not [17]. Based on the possibility distribution $\pi_{V}$ of $V$, the possibility and necessity that $A$ satisfies $C$ can be found. This proposition can thus be seen as a possibilistic variable on $\mathbb{B}$. The required possibility and necessity are:

$$
\begin{aligned}
& \left.\operatorname{Pos}(A \text { satisfies } C)=\min _{a \in A}\left(\sup _{(w, a) \in R} \pi_{V}(w)\right)\right) \\
& N e c(A \text { satisfies } C)=\min _{a \in A}\left(\inf _{(w, a) \notin R} 1-\pi_{V}(w)\right)
\end{aligned}
$$

Now, to calculate the possibility or necessity of a set $A$ satisfying multiple constraints, the min t-norm operator is used. For example:

$$
\begin{gathered}
\operatorname{Pos}\left(\left(A \text { satisfies } C_{1}\right) \text { and }\left(A \text { satisfies } C_{2}\right)\right)= \\
\min _{a \in A}\left(\operatorname{Pos}\left(A \text { satisfies } C_{1}\right), \operatorname{Pos}\left(A \text { satisfies } C_{2}\right)\right) \\
\operatorname{Nec}\left(\left(A \text { satisfies } C_{1}\right) \text { and }\left(A \text { satisfies } C_{2}\right)\right)= \\
\min _{a \in A}\left(\operatorname{Nec}\left(A \text { satisfies } C_{1}\right), \operatorname{Nec}\left(A \text { satisfies } C_{2}\right)\right)
\end{gathered}
$$

\section{Possibilistic Valid-Time Query Evaluation}

In this section, the proposal of this paper is presented. The proposal consists of an approach for querying a valid-time relation [10]. The novelty of this approach is that it allows uncertainty in the temporal demand in the query. To be able to present the query results to the user, this section also presents an approach for 
ranking the records in the queried relation, integrating the consequences of the query uncertainty into the ranking.

To illustrate the proposal of this paper, an example will be presented and examined in the course of the proposal presentation.

\subsection{The Valid-Time Relation}

An approach for querying a valid-time relation [10] is presented. Here, such a relation should contain one or more attributes, called valid-time attributes [10], specifying exactly one valid-time interval [10] for each record. This valid-time interval should be a crisp interval in the temporal domain, representing the uninterrupted period of time during which the object or concept represented by the corresponding record is real or true in the modelled reality.

An example relation is visualized in table 1. Every record represents a certain rental car owned by a car rental service, in a certain state. The attributes 'Startdate' and 'Enddate' describe the dates on which a time interval starts respectively ends (this interval includes the start- and enddates themselves) during which a car represented by a record, in the state represented by this record, is available for rent. The chronons are days. The attribute 'Mileage' describes the amount of kilometers the car has already driven. The attribute 'Color' describes the color of the car. Every unique value for the 'ID' attribute corresponds to a unique physical car. For the same car, every different value for attribute 'IID' corresponds to a different state of the car.

Table 1. An example relation containing car rental information. Mileage is in kilometers, temporal data is in $\mathrm{dd} / \mathrm{mm} /$ yyyy format.

\begin{tabular}{|c|c|c|c|c|}
\hline ID & III & Colo & Mileage & Startdate Enddate \\
\hline 001 & 1 & red & 20345 & $15 / 06 / 201214 / 0$ \\
\hline & 1 & blue & 23404 & 1210 \\
\hline 003 & 1 & blue & 25340 & 30/06/2012 30/08/2012 \\
\hline 004 & 1 & blue & 33367 & $01231 / 07$ \\
\hline 001 & 2 & red & 42420 & 15/08/2012 14/09/2012 \\
\hline
\end{tabular}

\subsection{The Query}

In this subsection, the proposed querying approach is presented. First, the query structure is presented, then the specific approach to query evaluation, followed by a ranking method.

Query Structure In the presented approach, the user's query demands may consist of several non-temporal demands and a single temporal demand. Nontemporal demands are demands concerning any attribute that is not a valid-time attribute, whereas the temporal demand concerns valid-time. The interpretation is that the user queries the relation for records satisfying both the non-temporal demands and the temporal demand. 
Thus, in this framework, every query $Q$ consists of two parts:

$$
Q=\left(Q_{T}, Q_{N}\right)
$$

Here, $Q_{N}$ denotes the collection of (possibly fuzzy) non-temporal user preferences. $Q_{T}$ denotes the temporal demand. A temporal demand $Q_{T}$ consists of two parts:

$$
Q_{T}=(A R, I K I)
$$

Here, $A R$ denotes an Allen relation [1] and $I K I$ denotes an ill-known interval in the valid-time domain used by the valid-time attributes of the relation. The interpretation of this temporal demand is that the user prefers a record which represents an object that is valid in the modelled reality during a time interval related to $I K I$. The nature of this relation is given by $A R$. The usage of an ill-known interval in the temporal demand allows for some uncertainty in the user's temporal preference: the user can query the relation searching for records representing objects valid during time intervals related by an Allen relation to a time interval that is partially unknown to the user.

As mentioned in [17], this approach differs from the one where a valid-time interval is represented by one fuzzy set. Such a fuzzy set is seen as a possibility distribution on some time domain $\mathbb{N}^{*}$ and thus defines just one ill-known value. However, in the presented approach, a time interval is represented by an illknown interval, which is defined by two possibility distributions on this $\mathbb{N}^{*}$, describing start and end point of the interval. As explained in section 2, this ill-known interval can be seen as defined by a possibility distribution on $\wp\left(\mathbb{N}^{*}\right)$.

An example is in order: consider a user who wants to rent a car during approximately the whole of july, preferably a blue one with a mileage below $30000 \mathrm{~km}$. Due to schedual issues and possible unforseen conditions, the user is not certain when exactly the rental car will be needed and thus on which exact day the rental car should start and end being available. These preferences can be translated into query $Q_{e x}$ :

$$
\begin{aligned}
Q_{e x} & =\left(Q_{T, e x}, Q_{N, e x}\right) \\
Q_{T, e x} & =\left(A R_{e x}, I K I_{e x}\right)
\end{aligned}
$$

Here, $Q_{N, e x}$ will denote that the car must be blue and have a mileage below $30000 \mathrm{~km} . A R_{e x}$ here denotes the Allen relation 'during'. Now to define $I K I_{e x}$, a definition of its start and end points are necessary. These start and end points are ill-known values, defined by possibilistic variables $S$ respectively $E$, which are defined by their possibility distributions $\pi_{S}$ respectively $\pi_{E}$. To define these possibility distributions, a translation of the notion 'approximately July' is necessary. This translation can be given by the user or suggested or constructed by the system. Imagine the user expressing that he will certainly not need the car on or before the 29th of June or on or after the 2nd of August. Also, the possibility that the user will need the car from the 1st of July to the 31th of July, boundaries included, is the highest. Lastly, the possibility that the user will need 
the car between the described periods varies linearly. The resulting possibility distributions are given by the equations below. In these equations, $\mathbb{T}$ is the set of all days in time, always denoted in dd/mm/yyyy format.

$$
\begin{aligned}
& \pi_{S}: \mathbb{T} \rightarrow[0,1] \\
& : x \rightarrow 0, \quad x \leq 29 / 06 / 12 \quad: x \rightarrow 1, \quad x \leq 31 / 07 / 12 \\
& : x \rightarrow 0.5, \quad x=30 / 06 / 12 \quad: x \rightarrow 0.5, \quad x=1 / 08 / 12 \\
& : x \rightarrow 1, \quad x \geq 1 / 07 / 12 \quad: x \rightarrow 0, \quad x \geq 2 / 08 / 12
\end{aligned}
$$

Query Evaluation The evaluation of a crisp query for a record in a regular (relational) database results in the accepting or rejecting of the record as a part of the result set presented to the user. In fuzzy querying, query satisfaction modelling is a matter of degree, as the evaluation of a fuzzy query for a record usually results in some satisfaction degree $s$, where $s \in[0,1]$, where 0 denotes total dissatisfaction and 1 denotes complete satisfaction. Now, the evaluation of a crisp query can also be modelled using similar satisfaction degrees, by assigning rejection a degree of 0 and acceptance a degree of 1 and not using any other value in $[0,1]$.

In this work, the evaluation of a query $Q=\left(Q_{T}, Q_{N}\right)$, with $Q_{T}=(A R, I K I)$ is handled as follows. For each record $r$ in the database, two things happen independently:

- The non-temporal preferences in $Q_{N}$ are evaluated. This results in a satisfaction degree $e_{Q_{N}}(r)$. The presented model accepts any valid and consistent way of calculating this evaluation, as long as $e_{Q_{N}}(r) \in[0,1]$.

- The temporal demand in $Q_{T}$ is evaluated. Depending on $A R$ and $I K I$, a specific set of ill-known constraints is constructed, which can be found in table 2. Based on equations (5) and (6) and using the min operator for aggregation, formulas are calculated to determine the possibility $\operatorname{Pos}_{Q^{T}}(r) \in$ $[0,1]$ and necessity $\operatorname{Nec}_{Q^{T}}(r) \in[0,1]$ that $r$ fulfills all these constraints. Because these formulas only depend on $A R$ and $I K I$, they only need to be constructed once and not for every considered record.

Table 2. The Allen relations with corresponding expressions for ill-known constraints. $A$ denotes a crisp interval. In the specification of the ill-known constraints, it is assumed that the corresponding ill-known interval $I K I$ is defined by a start point and an end point, respectively defined by possibilistic variables $\mathrm{S}$ and $\mathrm{E}$.

\begin{tabular}{c|c}
\hline Allen Relation & Constraints \\
\hline IKI before A & $C_{1} \triangleq(E,<)$ \\
\hline IKI equal A & $C_{1} \triangleq(S, \leq) \wedge C_{2} \triangleq(S, \neq) \wedge C_{3} \triangleq(E, \geq) \wedge C_{4} \triangleq(E, \neq)$ \\
\hline IKI meets A & $C_{1} \triangleq(E, \leq) \wedge \neg C_{2} \triangleq(E, \neq)$ \\
\hline IKI overlaps A & $C_{1} \triangleq(S,<) \wedge \neg C_{2} \triangleq(E, \leq) \wedge \neg C_{3} \triangleq(E, \geq)$ \\
\hline IKI during A & $\neg C_{1} \triangleq(S, \leq) \wedge \neg C_{2} \triangleq(E, \geq)$ \\
\hline IKI starts A & $C_{1} \triangleq(S, \leq) \wedge \neg C_{2} \triangleq(S, \neq) \wedge \neg C_{3} \triangleq(E, \geq)$ \\
\hline IKI finishes A & $C_{1} \triangleq(E, \geq) \wedge \neg C_{2} \triangleq(E, \neq) \wedge \neg C_{3} \triangleq(S, \leq)$ \\
\hline
\end{tabular}


Ranking As a final step, every record $r$ is given a final rank $e_{\text {final }}(r)$ depending on $e_{Q_{N}}(r)$ and a value $e_{Q_{T}}(r)$ based on both $\operatorname{Pos}_{Q^{T}}(r)$ and $N e c_{Q^{T}}(r)$. For every record $r$, this is done as follows.

First, $e_{Q_{T}}(r)$ is calculated using the expression in equation (9).

$$
e_{Q_{T}}(r)=\frac{\operatorname{Pos}_{Q^{T}}(r)+N e c_{Q^{T}}(r)}{2}
$$

Because necessity cannot exceed 0 unless possibility is 1 and $\operatorname{Pos}_{Q^{T}}(r) \in[0,1]$ and $N e c_{Q^{T}}(r) \in[0,1]$, the sum in the numerator gives a natural ranking score in $[0,2]$. The function of the denominator is to normalize this score to a value in $[0,1]$. The final ranking $e_{\text {final }}(r)$ is now given by a convex combination:

$$
e_{\text {final }}(r)=\omega * e_{Q_{N}}(r)+(1-\omega) * e_{Q_{T}}(r), \omega \in[0,1]
$$

The use of this convex combination allows a record to make up for a low score for the temporal constraint by a good score for the non-temporal constraint (or vice versa). Changing $\omega$ also allows granting the temporal constraint more weight with respect to the non-temporal constraint (or vice versa). The result of the evaluation and ranking steps on the example are shown in table 3. With $\omega=0.5$, it is clear that both non-temporal and temporal criteria have the same importance and the final ranking is natural.

Table 3. Scores and final ranking for the example records, using $\omega=0.5$.

\begin{tabular}{ccccccc}
\hline ID & IID & $e_{Q_{N}}(r)$ & $\operatorname{Pos}_{Q^{T}}(r)$ & $N e c_{Q^{T}}(r)$ & $e_{Q_{T}}(r)$ & $e_{\text {final }}(r)$ \\
\hline 001 & 1 & 0 & 1 & 1 & 1 & 0.5 \\
002 & 1 & 1 & 1 & 1 & 1 & 1 \\
003 & 1 & 1 & 0.5 & 0 & 0.25 & 0.625 \\
004 & 1 & 0 & 1 & 1 & 1 & 0.5 \\
001 & 2 & 0 & 0 & 0 & 0 & 0 \\
\hline
\end{tabular}

\section{Conclusions}

In this paper, a technique for querying valid-time relations is presented. This technique allows defining a temporal constraint based on a (partially) unknown time interval. The main advantage of this technique is that it tries to correctly represent and handle uncertainty in the temporal constraint, by using ill-known intervals. The technique allows the usage of both the Allen relations and more complex constructions. For future work, allowing the temporal specifications in the database to be (partially) unknown and new methods for aggregation and ranking are considered.

Acknowledgements. Part of the research is supported by grant BES-2009013805 within research project TIN2008-02066: Fuzzy Temporal Information Treatment in Relational DBMS. 


\section{References}

1. Allen, J.: Maintaining knowledge about temporal intervals. Communications of the ACM 26, 832-843 (1983)

2. Billiet, C., Pons, J.E., Matthé, T., De Tré, G., Pons Capote, O.: Bipolar fuzzy querying of temporal databases. In: Lecture Notes in Artificial Intelligence, vol. 7022, pp. 60-71. Springer, Ghent, Belgium (2011)

3. Bolour, A., Anderson, T.L., Dekeyser, L.J., Wong, H.K.T.: The role of time in information processing: a survey. ACM SIGMOD Record 12, 27-50 (1982)

4. Van der Cruyssen, B., De Caluwe, R., De Tré, G.: A theoretical fuzzy time model based on granularities. In: Proc. of the 5th European Congress on Intelligent Techniques and Soft Computing, pp. 1127-1131. ELITE Foundation (1997)

5. Devos, F., et al.: Modelling vague lexical time expressions by means of fuzzy set theory. Journal of Quantitative Linguistics 1(3), 189-194 (1994)

6. Dubois, D., HadjAli, A., Prade, H.: Fuzziness and uncertainty in temporal reasoning. Journal of Universal Computer Science 9(9), 1168-1194 (2003)

7. Dubois, D., Prade, H.: Incomplete conjunctive information. Computers \& Mathematics with Applications 15, 797-810 (1988)

8. Dubois, D., Prade, H.: Possibility Theory: An Approach to Computerized Processing of Uncertainty. Plenum Press, New York (1988)

9. Dubois, D., Prade, H.: Processing fuzzy temporal knowledge. Systems, Man and Cybernetics, IEEE Transactions on 19, 729-744 (1989)

10. Dyreson, C., Grandi, F., et al., W.K.: A consensus glossary of temporal database concepts. ACM SIGMOD Record 23, 52-64 (1994)

11. Garrido, C., Marín, N., Pons, O.: Fuzzy intervals to represent fuzzy valid time in a temporal relational database. International Journal of Uncertainty, Fuzziness and Knowledge-Based Systems 17(Supplementary Issue 1), 173-192 (2009)

12. Klein, W.: Time in Language. Routledge, London, U.K. (1994)

13. Mitra, D., et al.: A possibilistic interval constraint problem: Fuzzy temporal reasoning. In: Fuzzy Systems, 1994. IEEE World Congress on Computational Intelligence., Proc. of the Third IEEE Conference on, vol. 2, pp. 1434-1439 (1994)

14. Nagyp'al, G., Motik, B.: A fuzzy model for representing uncertain, subjective, and vague temporal knowledge in ontologies. In: On The Move to Meaningful Internet Systems 2003: CoopIS, DOA, and ODBASE, Lecture Notes in Computer Science, vol. 2888, pp. 906-923. Springer Berlin / Heidelberg (2003)

15. Nascimento, M., Eich, M.: Decision time in temporal databases. In: Proc. of the Second International Workshop on Temporal Representation and Reasoning, pp. 157-162 (1995)

16. Ohlbach, H.: Relations between fuzzy time intervals. Temporal Representation and Reasoning, International Syposium on 0, 44-51 (2004)

17. Pons, J.E., et al.: Possibilistic evaluation of sets (2011). Submitted to the International Journal of Uncertainty, Fuzziness and Knowledge-Based Systems

18. Schockaert, S., De Cock, M., Kerre, E.: Fuzzifying allen's temporal interval relations. Fuzzy Systems, IEEE Transactions on 16(2), 517-533 (2008)

19. Zadeh, L.: Fuzzy sets. Information and Control 8, 338-353 (1965) 